\title{
CHRONIC POLYARTHRITIS PRECEDING THE DIAGNOSIS OF WHIPPLE'S DISEASE - CASE REPORT
}

Juliana de Jesus Boscolo ${ }^{1, \star}$, Carlos Augusto Rodrigues Padilha ${ }^{1}$, Danielly Dantas Pimentel ${ }^{1}$, Gustavo Roberto Lourenço ${ }^{1}$, lane Tamara Dondé ${ }^{1}$, Maria Juliana da Silva Almeida ${ }^{1}$, Patrícia Milani de Moraes ${ }^{1}$, Renata Vaz de Oliveira ${ }^{1}$, Taísa Morete da Silva²

1.Faculdade de Medicina de São José do Rio Preto, São José do Rio Preto (SP), Brazil; 2.Hospital de Base, São José do Rio Preto (SP), Brazil.

*Corresponding author: julianaboscolo.jjb@gmail.com

\section{BACKGROUND}

Whipple's disease is a rare entity, classically characterized by joint symptoms, abdominal pain, weight loss, and diarrhea. Caused by Tropheryma whipplei, its clinical course is biphasic, with joint symptoms preceding diarrhea and weight loss by approximately six years. The joint involvement may manifest as polyarthritis or oligoarthritis, is more frequently intermittent and migratory, although it may present as chronic seronegative and destructive polyarthritis.

\section{CASE REPORT}

A 61-year-old female was admitted for the investigation of chronic diarrhea with signs of intestinal bleeding, emesis, and weight loss. The patient was a hypertensive former smoker with a history of chronic polyarthritis that began 11 years ago. It affected the metacarpophalangeal, proximal interphalangeal, wrist, ankle, and knee joints. This was associated with morning stiffness, elevated inflammatory markers, synovitis on physical examination, and negative rheumatoid factor and anti-cyclic citrullinated peptide. The patient was followed up with a rheumatology service for a presumptive diagnosis of seronegative rheumatoid arthritis. During the follow-up, she received methotrexate and leflunomide, followed by golimumab, tocilizumab, and abatacept. Except for golimumab, which was suspended due to therapeutic failure, all medications were replaced due to four episodes of consumptive syndrome, of which the last two were associated with diarrhea. Due to her diarrhea, melena, hematochezia, inappetence, emesis, and weight loss of about $20 \mathrm{~kg}$ (approximately 35\% of body weight), the patient was referred to the internal department for investigation. Laboratory testing revealed hypoalbuminemia, and normocytic and normochromic anemia. Chest computed tomography (CT) scan showed findings of centrilobular and paraseptal emphysema and bullae. The abdominal CT scan revealed thickening and parietal enhancement of the ileal loops and obliteration of the adjacent mesenteric fat. Colonoscopy showed lymphangitis in the terminal ileum. On endoscopy, endoscopic enanthematous gastritis, mild erosive gastritis of the antrum, and marked bulbar duodenitis were noted. Biopsy supported chronic duodenitis, ileitis, and colitis with an accumulation of macrophages in the lamina propria of the mucosa containing PAS-positive debris, compatible with Whipple's disease. Treatment was started with ceftriaxone followed by a schedule of sulfamethoxazole-trimethoprim for one year, with improvement in her condition.

\section{CONCLUSION}

Whipple's disease, although rare, should be suspected in patients with joint involvement, abdominal pain, weight loss, and diarrhea, or in cases of migratory seronegative polyarthritis unresponsive to immunosuppressive therapy.

\section{KEYWORDS}

Whipple's disease, Weight loss, Chronic polyarthritis. 